\title{
Anomalous enhancement of the isospin-violating $\Lambda(1405)$ production by a triangle singularity in $\Lambda_{c} \rightarrow \pi^{+} \pi^{0} \pi^{0} \Sigma^{0}$
}

\author{
L. R. Dai, ${ }^{1,2, *}$ R. Pavao, ${ }^{2, \dagger}$ S. Sakai, ${ }^{2, \$}$ and E. Oset ${ }^{2,}$ \\ ${ }^{1}$ Department of Physics, Liaoning Normal University, Dalian 116029, China \\ ${ }^{2}$ Departamento de Física Teórica and IFIC, Centro Mixto Universidad de Valencia-CSIC, \\ Institutos de Investigacion de Paterna, Apartado 22085, Valencia 46071, Spain
}

(Received 6 April 2018; published 5 June 2018)

\begin{abstract}
The decay of $\Lambda_{c}^{+}$into $\pi^{+} \pi^{0} \Lambda(1405)$ with the $\Lambda(1405)$ decay into $\pi^{0} \Sigma^{0}$ through a triangle diagram is studied. This process is initiated by $\Lambda_{c}^{+} \rightarrow \pi^{+} \bar{K}^{*} N$, and then the $\bar{K}^{*}$ decays into $\bar{K} \pi$ and $\bar{K} N$ produce the $\Lambda(1405)$ through a triangle loop containing $\bar{K}^{*} N \bar{K}$ which develops a singularity around $1890 \mathrm{MeV}$. This process is prohibited by the isospin symmetry, but the decay into this channel is enhanced by the contribution of the triangle diagram, which is sensitive to the mass of the internal particles. We find a narrow peak in the $\pi^{0} \Sigma^{0}$ invariant mass distribution, which originates from the $\Lambda(1405)$ amplitude, but is tied to the mass differences between the charged and neutral $\bar{K}$ or $N$ states. The observation of the unavoidable peak of the triangle singularity in the isospin-violating $\Lambda(1405)$ production would provide further support for the hadronic molecular picture of the $\Lambda(1405)$ and further information on the $\bar{K} N$ interaction.
\end{abstract}

DOI: 10.1103/PhysRevD.97.116004

\section{INTRODUCTION}

The possible role of a triangle singularity (TS) in hadronic reactions has been studied for a long time. The TS appears from a loop contribution in the decay of particle 1 into two particles 2 and 3 through the following process: at first particle 1 decays into particles $A$ and $B$, and particle $A$ subsequently decays into particles 2 and $C$, and finally particles $B$ and $C$ merge and form particle 3 in the final state. The TS was originally studied in Ref. [1], and it was found in Ref. [2] that the TS appears when the loop process has a classical counterpart; i.e., all the momenta of the particles in the loop (particles $A, B$, and $C$ in the above reaction) can be placed on-shell and the momenta of particles 2 and $B$ are antiparallel. A refined formulation based on Feynman diagrams and a simple formula for the position of the TS were given in Ref. [3]. One should note that the singularity would be smeared by the width of hadrons and appear as a broad peak in actual reactions. This peak purely comes from a kinematical

\footnotetext{
*dailr@1nnu.edu.cn

rpavao@ific.uv.es

*shuntaro.sakai@ific.uv.es

${ }^{\S}$ Eulogio.Oset@ific.uv.es
}

Published by the American Physical Society under the terms of the Creative Commons Attribution 4.0 International license. Further distribution of this work must maintain attribution to the author(s) and the published article's title, journal citation, and DOI. Funded by SCOAP. effect, and then we cannot associate this peak with a resonant state. In Refs. [4-6], it was found that the " $a_{1}(1420)$ " peak in the $\pi f_{0}(980)$ invariant mass distribution with a $p$-wave pion observed by COMPASS Collaboration [7] can be understood as a peak of a TS. In this process, the triangle diagram is formed by the $a_{1}(1260)$ decaying into $K \bar{K}^{*}\left(K^{*} \bar{K}\right)$ with a subsequent $\bar{K}^{*} \rightarrow \pi \bar{K}\left(K^{*} \rightarrow \pi K\right)$ decay and merging $K \bar{K}$ to form $f_{0}(980)$. The $a_{1}(1260)$ and the $f_{0}(980)$ have sizable couplings to the $K \bar{K}^{*}+$ c.c. and the $K \bar{K}$ channels, respectively, because they are dynamically generated through the coupled-channel effect of hadrons as studied in Refs. [8-10] and [11-17] for the $a_{1}(1260)$ and the $f_{0}(980)$, respectively. Actually, the large coupling of the internal particles and the final-state hadron is crucial to have a prominent peak of the TS. Nowadays, many hadronic molecular states have been studied as summarized in Ref. [18]. Because these states tend to have a large coupling to their constituent hadrons, the observation of the inevitable peak from the TS would provide further clues to clarify the nature of the hadronic molecules. Other than the " $a_{1}(1420)$ " the interpretation of the " $f_{1}(1420)$ " and " $f_{2}(1810)$ " in the PDG [19] as a peak of the triangle singularity was proposed in Refs. [20,21], respectively. Furthermore, possible manifestations of the TS in the heavy sector were investigated in Refs. [5,22-27].

On the other hand, it was found that the TS gives a significant contribution to the isospin-violating process. In Refs. [28-30], the role of the triangle diagram in the 
unusually large isospin-violating $\pi^{0} f_{0}(980)$ production from $\eta(1405)$ observed in BESIII [31] was studied. The triangular diagrams formed by $K^{*-} K^{+} K^{-}$and $\bar{K}^{* 0} K^{0} \bar{K}^{0}$ contribute to this process because of the sensitivity of the triangle singularity to the masses of the particles in the loop diagram, and the TS can have a sizable contribution in the isospin-violating process. It is noteworthy that the shape of the $f_{0}(980)$ resonance appears narrower than observed in other processes because the resonance shape is modified by the amplitude of the triangle diagram, which gives the width with the order of the charged- and neutral-kaon mass difference. Also, the line shape of the $\pi \pi$ invariant mass distribution calculated with the triangle diagram agrees with what was observed experimentally [31]. Following these studies, the isospin-violating $f_{0}(980)$ productions enhanced by the TS in the $D_{s}^{+} \rightarrow$ $\pi^{+} \pi^{0} f_{0}(980)$ and $\bar{B}_{s}^{0} \rightarrow J / \psi \pi^{0} f_{0}(980)$ processes were studied in Refs. [32,33], respectively.

In this paper, we focus on the isospin-violating $\Lambda_{c}^{+} \rightarrow$ $\pi^{+} \pi^{0} \Lambda(1405)$ process with the $\Lambda(1405)$ decay into $\pi^{0} \Sigma^{0}$ from the triangle diagram. The triangle diagram is formed by the decay of $\Lambda_{c}^{+}$into $\pi^{+} K^{*-} p\left(\pi^{+} \bar{K}^{* 0} n\right)$ followed by the decay of $K^{*-} \rightarrow \pi^{0} K^{-}\left(\bar{K}^{* 0} \rightarrow \pi^{0} \bar{K}^{0}\right)$ and the fusion of the $K^{-} p\left(\bar{K}^{0} n\right)$ to form $\Lambda(1405)$. From the formula of Eq. (18) in Ref. [3], a singularity from the triangle diagram would appear around $1890 \mathrm{MeV}$ in the $\pi^{0} \Lambda(1405)$ invariant mass distribution. The $\Lambda(1405)$ is successfully described as a hadronic molecule [34-42] and has a large coupling to the $\bar{K} N$ and the $\pi \Sigma$ channels (see also Refs. $[43,44]$ and references therein for details). The decay of heavy hadrons containing a charm or bottom quark is an exciting field in hadron physics as summarized in Ref. [45], and particularly the $\Lambda(1405)$ production in the $\Lambda_{c}^{+}, \chi_{c 0}(1 P)$, and $\Xi_{b}$ decays was studied in Refs. [46,47], [48], and [49], respectively, where the $\Lambda(1405)$ affects the $\pi \Sigma$ or $\bar{K} N$ mass distribution through the final-state rescattering. Considering the external $W^{+}$ emission for the transition of $\Lambda_{c}^{+}$into $\pi^{+} \bar{K}^{*} N$, which would give the main contribution to this process, the $\Lambda(1405)$ production is isospin forbidden. Indeed the $W$ produces the $\pi^{+}$in one vertex and in the other one includes a $c s$ transition. We have thus $\pi^{+}$and $s u d$, with $u d$ in $I=0$, because there these quarks are spectators. Thus the sud final state has $I=0$ and hadronizes in $\bar{K}^{*} N$ (see Fig. 3 below). Meanwhile, the possible effect of the TS on the $\Lambda(1405)$ production was studied in Refs. [50-52]. Now, as found in Refs. [28-30,32,33] for the $f_{0}(980)$ production, we expect that the isospinviolating $\Lambda(1405)$ production is enhanced by the TS around $1890 \mathrm{MeV}$ in the $\pi^{0} \Lambda(1405)$ mass distribution, where the triangle singularity would appear from the formula in Ref. [3], and that a narrow peak around the $\Lambda(1405)$ energy in the $\pi^{0} \Sigma^{0}$ mass distribution would appear. The observation of the TS in this isospin-violating $\Lambda(1405)$ production would give further support to the hadronic molecular picture of the $\Lambda(1405)$ resonance and provide us better understanding on the triangle singularity.

\section{FORMALISM}

In the present study, we investigate the $\Lambda_{c}^{+} \rightarrow \pi^{+} \pi^{0} \pi^{0} \Sigma^{0}$ decays via $\Lambda(1405)$ formation. The process of $\Lambda_{c}^{+} \rightarrow$ $\pi^{+} K^{*-} p$ followed by the $K^{*-}$ decay into $\pi^{0} K^{-}$and the merging of the $K^{-} p$ into $\Lambda(1405)$ [see Fig. 1(a)] or $\Lambda_{c}^{+} \rightarrow$ $\pi^{+} \bar{K}^{* 0} n$ followed by the $\bar{K}^{* 0}$ decay into $\pi^{0} \bar{K}^{0}$ and the merging of the $\bar{K}^{0} n$ into $\Lambda(1405)$ [see Fig. 1(b)] generate a singularity, and we will see a signal for the $\Lambda(1405)$ around $1420 \mathrm{MeV}$ because it comes from $\bar{K} N$ which couples to the second pole at $1420 \mathrm{MeV}$ in the invariant mass of $\pi^{0} \Sigma^{0}$. In the study of Ref. [38], the $\Lambda(1405)$ appears as the dynamically generated state of $K^{-} p, \bar{K}^{0} n, \pi^{0} \Lambda, \pi^{0} \Sigma^{0}, \eta \Lambda$, $\eta \Sigma^{0}, \pi^{+} \Sigma^{-}, \pi^{-} \Sigma^{+}, K^{+} \Xi^{-}$, and $K^{0} \Xi^{0}$ in the coupledchannels calculation.

We will analyze the effect of triangle singularities in the decay of $\Lambda_{c}^{+} \rightarrow \pi^{+} \pi^{0} \pi^{0} \Sigma^{0}$. In this study, we focus on the decay channel of $\pi^{0} \Sigma^{0}$ from $\Lambda(1405)$, which does not contain the $I=1$ contribution and has a small $I=2$ one, to focus on the isospin violation. The complete Feynman diagram for the decay with the triangle mechanism through the $\Lambda(1405)$ baryon is shown in Fig. 1, and the momenta assignment for the decay process is given in Fig. 2. In the hadronization of Fig. 3(b) we shall see in the next subsection that $\bar{K}^{*} N$ is produced. The $\bar{K}^{*}$ will decay into $\pi \bar{K}$, and thus the triangular mechanisms of Fig. 1 appear.

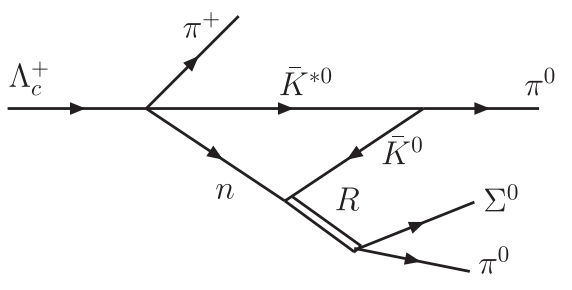

(b)

FIG. 1. Diagram for the decay of $\Lambda_{c}^{+} \rightarrow \pi^{+} \pi^{0} \pi^{0} \Sigma^{0}$. (a) The process of $\Lambda_{c}^{+} \rightarrow \pi^{+} K^{*-} p$ followed by the $K^{*-}$ decay into $\pi^{0} K^{-}$and the merging of the $K^{-} p$ into $\Lambda(1405)$; (b) $\Lambda_{c}^{+} \rightarrow \pi^{+} \bar{K}^{* 0} n$ followed by the $\bar{K}^{* 0}$ decay into $\pi^{0} \bar{K}^{0}$ and the merging of the $\bar{K}^{0} n$ into $\Lambda(1405)$. 


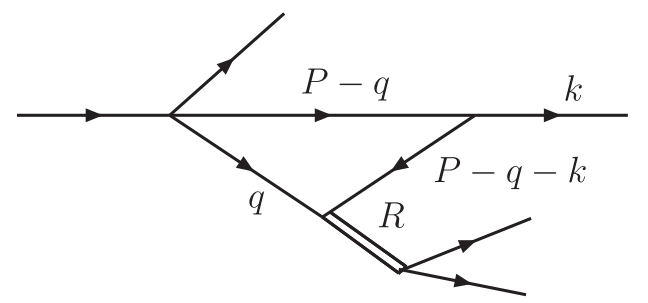

FIG. 2. The momenta assignment for the decay process.

Now we would like to evaluate the $\Lambda_{c}^{+} \rightarrow \pi^{+} \pi^{0} R$ with the $R \rightarrow \pi^{0} \Sigma^{0}$ process which produces the triangle diagram shown in Fig. 1, where $R$ stands for the $\Lambda(1405)$ resonance.

First, let us consider the $T$ matrix element $t$ for the triangle loop process shown in Fig. 1(a), which, following Feynman rules (see also Ref. [3] for further details), is given by

$$
\begin{aligned}
t= & i \sum_{\text {polof } K^{*}} 2 M_{p} \int \frac{d^{4} q}{(2 \pi)^{4}} \frac{t_{K^{-} p \rightarrow \pi^{0} \Sigma^{0}}}{q^{2}-M_{p}^{2}+i \epsilon} \\
& \times \frac{t_{K^{*-} \rightarrow \pi^{0} K^{-}}}{(P-q)^{2}-m_{K^{*-}}^{2}+i \epsilon} \frac{t_{\Lambda_{c}^{+} \rightarrow \pi^{+} K^{*-} p}}{(P-q-k)^{2}-m_{K^{-}}^{2}+i \epsilon},
\end{aligned}
$$

where we use the field normalization of Mandl and Shaw [53]. The amplitude in Eq. (1) is evaluated in the center-ofmass (CM) frame of $\pi^{0} R$. Thus we need to calculate the three vertices, $t_{\Lambda_{c}^{+} \rightarrow \pi^{+} K^{*-} p}, t_{K^{*-} \rightarrow \pi^{0} K^{-}}$, and $t_{K^{-} p \rightarrow \pi^{0} \Sigma^{0}}$, in Eq. (1).

\section{A. Decay mechanism at quark level}

Looking at the $\Lambda_{c}^{+}$decay mechanism depicted in Fig. 3(a), at the quark level the Cabibbo-allowed vertex is formed through an external emission of a $W$ boson [54], which is also color-favored, producing a $u \bar{d}$ pair that forms the $\pi^{+}$. Note that $u d$ in the $\Lambda_{c}^{+}$are in $I=0$, and since they are spectators in the reaction they also have $I=0$ in the final state of Fig. 3(a). This, added to the $s$ quark that has no isospin, gives us $I=0$ for the final baryon before the hadronization, and this continues to be the case after the hadronization which is based on strong interaction. The hadronization proceeds via $\bar{u} u+\bar{d} d+\bar{s} s$ pair creation with the quantum numbers of the vacuum on top of the sud state [see Fig. 3(b)]. The resulting meson and baryon from the hadronization of Fig. 3(b) are easily obtained by writing

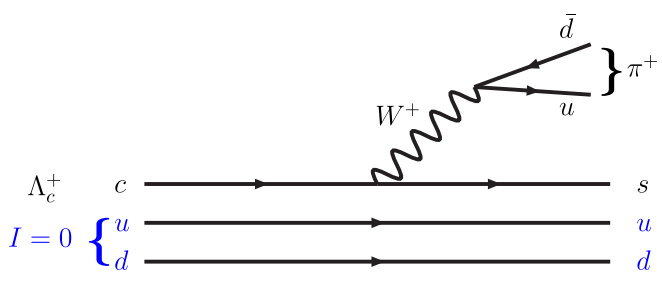

(a)

$$
H=\sum_{i=1}^{3} s \bar{q}_{i} q_{i} \frac{1}{\sqrt{2}}(u d-d u)=\sum_{i=1}^{3} M_{3 i} q_{i} \frac{1}{\sqrt{2}}(u d-d u),
$$

where $M_{i j}$ are the components of the $S U(3)_{f} q \bar{q}$ matrix with the $u, d, s$ quarks,

$$
M=\left(\begin{array}{ccc}
u \bar{u} & u \bar{d} & u \bar{s} \\
d \bar{u} & d \bar{d} & d \bar{s} \\
s \bar{u} & s \bar{d} & s \bar{s}
\end{array}\right) .
$$

However, as we are interested in $\bar{K}^{*} N$ production for the triangle singularity, we are only concerned at this point about the weights of the different flavor states. To implement that we write the flavor composition of the vector states:

$$
\begin{array}{rlrl}
\rho^{0} & =\frac{1}{\sqrt{2}}(u \bar{u}-d \bar{d}), & \omega=\frac{1}{\sqrt{2}}(u \bar{u}+d \bar{d}), \\
\rho^{+} & =u \bar{d}, & \rho^{-}=d \bar{u}, & K^{* 0}=d \bar{s}, \\
K^{*-} & =s \bar{u}, & K^{*+}=u \bar{s}, & \bar{K}^{* 0}=s \bar{d}, \\
\phi & =s \bar{s} . & &
\end{array}
$$

Thus we can write the $S U(3)_{f} q \bar{q}$ matrix in terms of physical vector mesons as

$$
M \rightarrow V=\left(\begin{array}{ccc}
\frac{\rho^{0}}{\sqrt{2}}+\frac{\omega}{\sqrt{2}} & \rho^{+} & K^{*+} \\
\rho^{-} & -\frac{\rho^{0}}{\sqrt{2}}+\frac{\omega}{\sqrt{2}} & K^{* 0} \\
K^{*-} & \bar{K}^{* 0} & \phi
\end{array}\right) .
$$

Then we get

$$
\begin{aligned}
H= & K^{*-} u \frac{1}{\sqrt{2}}(u d-d u)+\bar{K}^{* 0} d \frac{1}{\sqrt{2}}(u d-d u) \\
& +\phi s \frac{1}{\sqrt{2}}(u d-d u) .
\end{aligned}
$$

By looking at the quark content of the octet baryons in Ref. [55] (see Table III of that work), we see that, in terms of three quarks, the mixed antisymmetric representation of

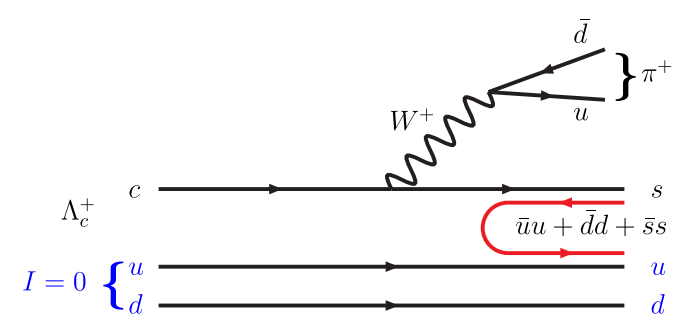

(b)

FIG. 3. (a) Diagram for $\Lambda_{c}^{+} \rightarrow \pi^{+} \pi^{0} \pi^{0} \Sigma^{0}$ decay. (b) Hadronization through $\bar{q} q$ creation with vacuum quantum numbers. 
the baryons, with phases compatible with the structure of the chiral Lagrangians gives

$$
\begin{aligned}
p & =\frac{1}{\sqrt{2}} u(u d-d u), \\
n & =\frac{1}{\sqrt{2}} d(u d-d u), \\
\Lambda & =\frac{1}{2 \sqrt{3}}[u(d s-s d)+d(s u-u s)-2 s(u d-d u)], \\
\Sigma^{0} & =\frac{1}{2}[u(d s-s d)-d(s u-u s)] .
\end{aligned}
$$

Hence, we can write the flavor content of $H$ as

$$
H=K^{*-} p+\bar{K}^{* 0} n-\sqrt{\frac{2}{3}} \phi \Lambda .
$$

However, we will neglect the $\phi \Lambda$ component since this does not contribute to our triangle singularity mechanism. Note that $s \frac{1}{\sqrt{2}}(u d-d u)$ has zero overlap with $\Sigma^{0}$ and thus the $\phi \Sigma^{0}$ component does not appear, as it should be, since this has $I=1$.

\section{B. Calculation of the three vertices}

\section{First vertex}

As usual, we take the structure for the transitions that involves the lowest orbital angular momentum, and since the $\Lambda_{c}^{+} \rightarrow \pi^{+} K^{*-} p$ process can proceed via $s$-wave, the amplitude for $t_{\Lambda_{c}^{+} \rightarrow \pi^{+} K^{*-} p}$ is given by

$$
t_{\Lambda_{c}^{+} \rightarrow \pi^{+} K^{*-p}}=A \vec{\sigma} \cdot \vec{\epsilon},
$$

where a scalar function is made between the spin and the polarization of the $\bar{K}^{*}$, since it cannot depend on momentum in the $s$-wave.

The $K^{*-} p$ invariant mass distribution of the $\Lambda_{c}^{+} \rightarrow$ $\pi^{+} K^{*-} p$ decay is easily obtained in this case as

$$
\begin{aligned}
\frac{d \Gamma_{\Lambda_{c}^{+} \rightarrow \pi^{+} K^{*-} p}}{d M_{\mathrm{inv}}\left(K^{*-} p\right)}= & \frac{1}{(2 \pi)^{3}} \frac{2 M_{\Lambda_{c}^{+}} 2 M_{p}}{4 M_{\Lambda_{c}^{+}}^{2}} p_{\pi^{+}} \tilde{p}_{K^{*-}} \\
& \times \sum \sum\left|t_{\Lambda_{c}^{+} \rightarrow \pi^{+} K^{*-} p}\right|^{2},
\end{aligned}
$$

where $p_{\pi^{+}}$is the momentum of $\pi^{+}$in the $\Lambda_{c}^{+}$rest frame, and $\tilde{p}_{K^{*-}}$ is the momentum of $K^{*-}$ in the $K^{*-} p$ rest frame,

$$
\begin{aligned}
p_{\pi^{+}} & =\frac{\lambda^{1 / 2}\left(M_{\Lambda_{c}^{+}}^{2}, m_{\pi}^{2}, M_{\mathrm{inv}}^{2}\left(K^{*-} p\right)\right)}{2 M_{\Lambda_{c}}}, \\
\tilde{p}_{K^{*-}} & =\frac{\lambda^{1 / 2}\left(M_{\mathrm{inv}}^{2}\left(K^{*-} p\right), m_{K^{*-}}^{2}, M_{p}^{2}\right)}{2 M_{\mathrm{inv}}\left(K^{*-} p\right)},
\end{aligned}
$$

with $\lambda(x, y, z)$ the ordinary Källen function. $\overline{\sum \sum|t|^{2}}$ symbolizes the average over the initial polarizations and sum over the final polarizations of $|t|^{2}$, which, using Eq. (8), is given by

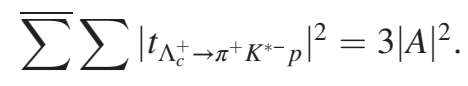

Since $\sum \sum|t|^{2}$ has no angular structure, one can follow the steps of Ref. [53] for $\mu \rightarrow \nu_{\mu} e^{-} \bar{\nu}_{e}$ decay to write the differential decay width as given by Eq. (9) (see also Ref. [16]).

By integrating Eq. (9) over $M_{\text {inv }}\left(K^{*-} p\right)$ and using Eq. (11) we obtain

$$
\frac{|A|^{2}}{\Gamma_{\Lambda_{c}^{+}}}=\frac{\operatorname{Br}\left(\Lambda_{c}^{+} \rightarrow \pi^{+} K^{*-} p\right)}{\int_{m_{K^{*-}}+M_{p}}^{M_{\Lambda^{+}-m^{+}}} \frac{3}{(2 \pi)^{3}} \frac{M_{p}}{M_{\Lambda_{c}^{+}}} p_{\pi^{+}} \tilde{p}_{K^{*-}} d M_{\mathrm{inv}}\left(K^{*-} p\right)} .
$$

By calculating the width of this decay, using the experimental branching ratio of this decay $\operatorname{Br}\left(\Lambda_{c}^{+} \rightarrow\right.$ $\left.\pi^{+} K^{*-} p\right)=(1.5 \pm 0.5) \times 10^{-2}$ [19], we can determine the value of the constant $|A|$.

\section{Second vertex}

Now we calculate the contribution of the vertex $K^{*-} \rightarrow$ $\pi^{0} K^{-}$by using the chiral invariant Lagrangian with local hidden symmetry given in Refs. [56-59], which is

$$
\mathcal{L}_{V P P}=-i g\left\langle V^{\mu}\left[P, \partial_{\mu} P\right]\right\rangle .
$$

The brackets $\langle\cdots\rangle$ mean the trace over the $\mathrm{SU}(3)$ flavor matrices, and the coupling is given by $g=m_{V} / 2 f_{\pi}$ in the local hidden gauge, with $m_{V}=780 \mathrm{MeV}$ and $f_{\pi}=$ $93 \mathrm{MeV}$. The $S U(3)_{f} q \bar{q}$ matrix written in terms of pseudoscalar mesons is given by

$$
M \rightarrow P=\left(\begin{array}{ccc}
\frac{\pi^{0}}{\sqrt{2}}+\frac{\eta}{\sqrt{3}}+\frac{\eta^{\prime}}{\sqrt{6}} & \pi^{+} & K^{+} \\
\pi^{-} & -\frac{\pi^{0}}{\sqrt{2}}+\frac{\eta}{\sqrt{3}}+\frac{\eta^{\prime}}{\sqrt{6}} & K^{0} \\
K^{-} & \bar{K}^{0} & -\frac{\eta}{\sqrt{3}}+\frac{\eta^{\prime}}{\sqrt{6}}
\end{array}\right),
$$

where the standard mixing of $\eta$ and $\eta^{\prime}$ has been assumed [60]. Performing the matrix operations and the trace we get for $K^{*-} \rightarrow \pi^{0} K^{-}$,

$$
\mathcal{L}_{K^{*-} \pi^{0} K^{-}}=-i g \frac{1}{\sqrt{2}} K^{*-\mu}\left(\pi^{0} \partial_{\mu} K^{+}-\partial_{\mu} \pi^{0} K^{+}\right) .
$$

Applying the Feynman rules derived from the Lagrangian of Eq. (15), for the $T$ matrix element we get 


$$
\begin{aligned}
-i t_{K^{*-} \rightarrow \pi^{0} K^{-}} & =i g \frac{1}{\sqrt{2}} \epsilon_{K^{*-}}^{\mu}\left(p_{K^{-}}-p_{\pi^{0}}\right)_{\mu} \\
& \simeq i g \frac{1}{\sqrt{2}} \vec{\epsilon}_{K^{*-}} \cdot\left(\vec{p}_{\pi^{0}}-\vec{p}_{K^{-}}\right),
\end{aligned}
$$

with $\vec{p}_{K^{-}}$and $\vec{p}_{\pi^{0}}$ calculated in the CM frame of $\pi^{0} R$. At the energy where the triangle singularity appears, the momentum of $K^{*-}$ is small enough, and one can omit the zeroth component of the polarization vector in Eq. (16). This is shown in detail in Appendix A of Ref. [27] where keeping the $\epsilon^{0}$ term of the vector polarization of the vector meson gives a correction of order $\left(\frac{p_{v}}{m_{v}}\right)^{2}$ with a coefficient that renders this term really small. In our case if we take $M_{\text {inv }}\left(K^{*-} p\right) \sim 1850 \mathrm{MeV}$, where the singularity appears, we get an effect of $0.5 \%$. If we take $M_{\text {inv }}\left(K^{*-} p\right)=$ $1900 \mathrm{MeV}$, the correction amounts to $2 \%$.

Similarly we get

$$
-i t_{K^{* 0} \rightarrow \pi^{0} \bar{K}^{0}} \simeq-i g \frac{1}{\sqrt{2}} \vec{\epsilon}_{K^{* 0}} \cdot\left(\vec{p}_{\pi^{0}}-\vec{p}_{\bar{K}^{0}}\right)
$$

which has opposite sign to Eq. (16).

\section{Third vertex}

The third vertex corresponds to the mechanism for the production of the $\pi^{0} \Sigma^{0}$ pair in the final state, after the rescattering of the $K^{-} p$ that dynamically generates the $\Lambda(1405)$ resonance as an intermediate state. We will write the vertex as

$$
t_{3} \equiv t_{K^{-} p \rightarrow \pi^{0} \Sigma^{0},}
$$

where $t_{i \rightarrow f}$ is the scattering matrix element between the initial state $i$ and the final state $f$ in the coupled channel calculation with the channels $K^{-} p(1), \bar{K}^{0} n$ (2), $\pi^{0} \Lambda$ (3), $\pi^{0} \Sigma^{0}$ (4), $\eta \Lambda$ (5), $\eta \Sigma^{0}$ (6), $\pi^{+} \Sigma^{-} \quad$ (7), $\pi^{-} \Sigma^{+}$ (8), $K^{+} \Xi^{-}$(9), and $K^{0} \Xi^{0}$ (10). We have $i=1$ for the diagrams of Fig. 1(a), while the index $f$ stands for channel 4. The $T$ matrix is obtained using the Bethe-Salpeter equation, with the tree level potentials given in Ref. [38],

$$
T=[1-V G]^{-1} V,
$$

where $V_{i j}$ are the potentials given in [38] and $G_{l}$ is the meson-baryon loop function for the intermediate states, which is diagonal. The loop functions $G_{l}$ for the intermediate states are regularized using the cutoff method, and the peak of the $\Lambda(1405)$ is well reproduced using a cutoff of $630 \mathrm{MeV}$. We will need this cutoff parameter for the next steps of the calculation, being necessary in order to evaluate the loop integral in the diagram of Fig. 1. We also need the amplitude $t_{3} \equiv t_{\bar{K}^{0} n \rightarrow \pi^{0} \Sigma^{0}}$ which corresponds to the matrix element $t_{24}$ of the coupled channel problem. In Fig. 4 we show amplitudes of $t_{K^{-} p \rightarrow \pi^{0} \Sigma^{0}}$ and $t_{\bar{K}^{0} n \rightarrow \pi^{0} \Sigma^{0}}$ as a function of the $\bar{K} N$ invariant mass.

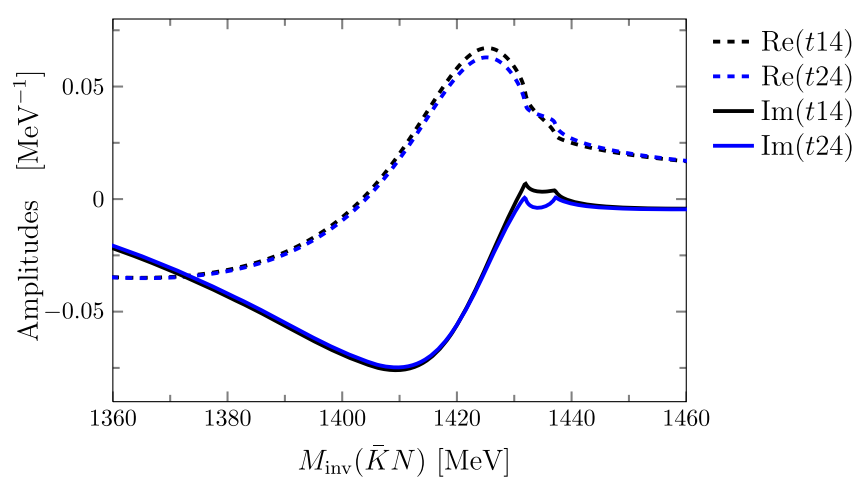

FIG. 4. $t_{K^{-} p \rightarrow \pi^{0} \Sigma^{0}}\left(t_{14}\right)$ and $t_{\bar{K}^{0} n \rightarrow \pi^{0} \Sigma^{0}}\left(t_{24}\right)$ evaluated with ten channels of Ref. [38]; dashed lines: real part; solid lines: imaginary part.

\section{The total amplitude}

Now we obtain the final amplitude of $\Lambda_{c}^{+} \rightarrow \pi^{+} \pi^{0} \pi^{0} \Sigma^{0}$ for Fig. 1(a), which makes more explicit the amplitude of Eq. (1),

$$
t_{\Lambda_{c}^{+} \rightarrow \pi^{+} \pi^{0} \pi^{0} \Sigma^{0}}=-A \frac{1}{\sqrt{2}} g \vec{\sigma} \cdot \vec{k} t_{K^{-} p \rightarrow \pi^{0} \Sigma^{0}} t_{T},
$$

where for simplicity we use $t_{T} \equiv t_{T}\left(m_{K^{*-}}, M_{p}, m_{K^{-}}\right)$for the triangle loop function for the decay shown in Fig. 1(a), defined as

$$
\begin{aligned}
t_{T}= & i \int \frac{d^{4} q}{(2 \pi)^{4}} \frac{2 M_{p}}{q^{2}-M_{p}^{2}+i \epsilon} \frac{1}{(P-q)^{2}-m_{K^{*-}}^{2}+i \epsilon} \\
& \times \frac{1}{(P-q-k)^{2}-m_{K^{-}}^{2}+i \epsilon}\left(2+\frac{\vec{q} \cdot \vec{k}}{\vec{k}^{2}}\right),
\end{aligned}
$$

where the term $\vec{q} \cdot \vec{k} / \vec{k}^{2}$ comes from the term proportional to $\vec{q}$ in the integrand. Indeed, Eq. (1) by means of Eqs. (8) and (17) gives the factor $\sigma_{i} \epsilon_{i} \epsilon_{j}\left(p_{\pi^{0}}-p_{K^{-}}\right)_{j}$, which upon summing over the $K^{*-}$ intermediate polarizations leads to $\sigma_{i}\left(p_{\pi^{0}}-p_{K^{-}}\right)_{i}$. According to Fig. 2, this gives us the factor $\sigma_{i}[\vec{k}-(\vec{P}-\vec{q}-\vec{k})]_{i}=\sigma_{i}(2 \vec{k}+\vec{q})_{i}$ since $\vec{P}=0$ in the $\pi^{0} R$ rest frame where we evaluate the amplitude. $\vec{k}$ is external, i.e., is not integrated over, and goes out of integrand, and for the $\vec{q}$ term we use (after performing the $q^{0}$ integral analytically)

$$
\int d^{3} q q_{i} \cdots \cdots=B k_{i},
$$

since the integral behaves as a vector under rotations, and after the $\vec{q}$ integrations the only vector that remains is $\vec{k}$. Multiplying by $k_{i}$ we get $\int d^{3} q q_{i} k_{i} \cdots \cdots=\int d^{3} q \vec{q}$. $\vec{k} \cdots \cdots=B \vec{k}^{2}$, which gives us the coefficient $B$ in Eq. (22). The analytical integration of $t_{T}$ over $q^{0}$ leads to $[3,61]$ 


$$
\begin{aligned}
t_{T}= & \int \frac{d^{3} q}{(2 \pi)^{3}} \frac{2 M_{p}}{8 \omega_{K^{*-}} \omega_{p} \omega_{K^{-}}} \frac{1}{k^{0}-\omega_{K^{-}}-\omega_{K^{*-}}+i \frac{\Gamma_{K^{*-}}}{2}} \\
& \times \frac{1}{P^{0}+\omega_{p}+\omega_{K^{-}}-k^{0}}\left(2+\frac{\vec{q} \cdot \vec{k}}{\vec{k}^{2}}\right) \\
& \times \frac{1}{P^{0}-\omega_{p}-\omega_{K^{-}}-k^{0}+i \epsilon} \\
& \times \frac{2 P^{0} \omega_{p}+2 k^{0} \omega_{K^{-}}-2\left(\omega_{p}+\omega_{K^{-}}\right)\left(\omega_{p}+\omega_{K^{-}}+\omega_{K^{*-}}\right)}{P^{0}-\omega_{K^{*-}}-\omega_{p}+i \frac{\Gamma_{K^{*-}}}{2}},
\end{aligned}
$$

with $P^{0}=M_{\mathrm{inv}}\left(\pi^{0} \Lambda(1405)\right), \omega_{p}=\sqrt{\vec{q}^{2}+M_{p}^{2}}, \omega_{K^{-}}=$ $\sqrt{(\vec{q}+\vec{k})^{2}+m_{K^{-}}^{2}}$, and $\omega_{K^{*-}}=\sqrt{\vec{q}^{2}+m_{K^{*-}}^{2}}$. The energy $k^{0}$ and momentum $|\vec{k}|$ of $\pi^{0}$ emitted from $\bar{K}^{*}$ are given by

$$
\begin{aligned}
k^{0} & =\frac{M_{\mathrm{inv}}^{2}\left(\pi^{0} \Lambda(1405)\right)+m_{\pi^{0}}^{2}-M_{\mathrm{inv}}^{2}\left(\pi^{0} \Sigma^{0}\right)}{2 M_{\mathrm{inv}}\left(\pi^{0} \Lambda(1405)\right)}, \\
|\vec{k}| & =\frac{\lambda^{1 / 2}\left(M_{\mathrm{inv}}^{2}\left(\pi^{0} \Lambda(1405)\right), m_{\pi^{0}}^{2}, M_{\mathrm{inv}}^{2}\left(\pi^{0} \Sigma^{0}\right)\right)}{2 M_{\mathrm{inv}}\left(\pi^{0} \Lambda(1405)\right)} .
\end{aligned}
$$

Following the method of Ref. [25], we obtain the final differential distribution for four particles in the final state,

$$
\begin{aligned}
& \frac{1}{\Gamma_{\Lambda_{c}^{+}}} \frac{d^{2} \Gamma}{d M_{\mathrm{inv}}\left(\pi^{0} \Lambda(1405)\right) d M_{\mathrm{inv}}\left(\pi^{0} \Sigma^{0}\right)} \\
& =\frac{1}{(2 \pi)^{5}} \frac{M_{\Sigma^{0}}}{M_{\Lambda_{c}^{+}}} \tilde{p}_{\pi^{+}} \tilde{p}_{\pi^{0}} \tilde{q}_{\Sigma^{0}} \frac{1}{2} g^{2} \frac{A^{2}}{\Gamma_{\Lambda_{c}^{+}}}|\vec{k}|^{2}\left|t_{T}\right|^{2} \\
& \quad \times\left|t_{K^{-} p \rightarrow \pi^{0} \Sigma^{0}}\right|^{2},
\end{aligned}
$$

with

$$
\begin{aligned}
& \tilde{p}_{\pi^{+}}=\frac{\lambda^{1 / 2}\left(M_{\Lambda_{c}^{+}}^{2}, M_{\mathrm{inv}}^{2}\left(\pi^{0} \Lambda(1405)\right), m_{\pi^{+}}^{2}\right)}{2 M_{\Lambda_{c}^{+}}}, \\
& \tilde{p}_{\pi^{0}}=|\vec{k}|=\frac{\lambda^{1 / 2}\left(M_{\mathrm{inv}}^{2}\left(\pi^{0} \Lambda(1405)\right), m_{\pi^{0}}^{2}, M_{\mathrm{inv}}^{2}\left(\pi^{0} \Sigma^{0}\right)\right.}{2 M_{\mathrm{inv}}\left(\pi^{0} \Lambda(1405)\right)},
\end{aligned}
$$

$$
\tilde{q}_{\Sigma^{0}}=\frac{\lambda^{1 / 2}\left(M_{\mathrm{inv}}^{2}\left(\pi^{0} \Sigma^{0}\right), m_{\pi^{0}}^{2}, M_{\Sigma^{0}}^{2}\right)}{2 M_{\mathrm{inv}}\left(\pi^{0} \Sigma^{0}\right)} .
$$

To regularize the integral in Eq. (23) we use the same cutoff of the meson loop that is used to calculate $t_{K^{-} p \rightarrow \pi^{0} \Sigma^{0}}$ in Eq. (18) with $\theta\left(q_{\max }-\left|\vec{q}^{*}\right|\right)$, where $\vec{q}^{*}$ is the $\vec{q}$ momentum in the $R$ rest frame (see Ref. [3]).

\section{Isospin-breaking effect}

If we use the same masses for $K^{-}, \bar{K}^{0}$, for $K^{*-}, \bar{K}^{* 0}$, and for $p$ and $n$ with isospin conservation, we find that the contributions from Figs. 1(a) and 1(b) will cancel each other. Indeed, as we discussed earlier the $K^{*-} p$ and $\bar{K}^{* 0} n$, coming from an original state of $s$ and $u d$ with $I=0$, have $I=0$. Using Clebsch-Gordan coefficients the vertices for $K^{*-} p$ or $\bar{K}^{* 0} n$ have opposite signs, but $K^{*-}$ is the isospin state $-|1 / 21 / 2\rangle$ and then the vertices are equal in consistency with Eq. (7). We also saw in Eqs. (16) and (17) that the $K^{*-} \rightarrow \pi^{0} K^{-}$and $\bar{K}^{* 0} \rightarrow \pi^{0} \bar{K}^{0}$ vertices have opposite signs. Finally, since the chiral Lagrangians are isospin symmetric, the scattering matrices are also isospin symmetric if the masses of mesons and baryons in the same isospin multiplets are equal. Then $\bar{K} N \rightarrow \pi^{0} \Sigma^{0}$ has only $I=0$, and once again the $p K^{-} \rightarrow \pi^{0} \Sigma^{0}$ and $n \bar{K}^{0} \rightarrow \pi^{0} \Sigma^{0}$ amplitudes have the same sign under isospin invariance once the phase of $K^{-} \equiv-|1 / 21 / 2\rangle$ is considered. This shows technically how the two diagrams of Fig. 1 cancel with exact isospin symmetry, and this should be the case because for $\pi^{0}+\Sigma^{0}$ one has $I=1$, but we started from an $I=0$ state. In the chiral unitary approach there is isospin violation due to the different masses of particles within the same isospin multiplets [38] and in studies of $f_{0}-a_{0}$ mixing in $J / \psi \rightarrow \phi \pi^{0} \eta$, it is again the difference of mass between $K^{0}$ and $K^{+}$in the loops that leads to the isospin violation [62-65].

Therefore, it is interesting to investigate the isospinbreaking effect. That means, for the first time, we will precisely look at the $\Lambda(1405)$ formation in an isospin forbidden mode. We expect that the formation will be driven by a triangle singularity and the shape will be narrower than usual, because it will be tied to the different masses of $\bar{K} N$. Therefore, in the following, we will use different masses for $K^{-} p$ or $\bar{K}^{0} n$, and also for $K^{*-}$ and $\bar{K}^{* 0}$.

Now we consider Fig. 1(b), for the $\Lambda_{c}^{+} \rightarrow \pi^{+} \bar{K}^{* 0} n$ followed by $\bar{K}^{* 0} \rightarrow \pi^{0} \bar{K}^{0}$ decay and $\bar{K}^{0} n \rightarrow \pi^{0} \Sigma^{0}$ to see the $\Lambda(1405)$ formation. We also need to calculate the three vertices, $t_{\Lambda_{c}^{+} \rightarrow \pi^{+} \bar{K}^{\star 0} n}, t_{\bar{K}^{\star 0} \rightarrow \pi^{0} \bar{K}^{0}}$, and $t_{\bar{K}^{0} n \rightarrow \pi^{0} \Sigma^{0}}$.

The triangle amplitude for the $\bar{K}^{* 0} n \bar{K}^{0}$ loop, $t_{T}=t_{T}\left(m_{\bar{K}^{* 0}}, M_{n}, m_{\bar{K}^{0}}\right)$, is obtained with Eq. (23) replacing the masses and width of the internal particles.

Hence, for the isospin-breaking effect, we get the final differential distributions,

$$
\begin{aligned}
& \frac{1}{\Gamma_{\Lambda_{c}^{+}}} \frac{d^{2} \Gamma}{d M_{\mathrm{inv}}\left(\pi^{0} \Lambda(1405)\right) d M_{\mathrm{inv}}\left(\pi^{0} \Sigma^{0}\right)} \\
& =\frac{1}{(2 \pi)^{5}} \frac{M_{\Sigma^{0}}}{M_{\Lambda_{c}^{+}}} \tilde{p}_{\pi^{+}} \tilde{q}_{\Sigma^{0}} \frac{1}{2} g^{2} \frac{A^{2}}{\Gamma_{\Lambda_{c}^{+}}}|\vec{k}|^{3} \\
& \quad \times \mid t_{T}\left(m_{K^{*-}}, M_{p}, m_{K^{-}}\right) t_{K^{-} p \rightarrow \pi^{0} \Sigma^{0}}-t_{T} \\
& \quad \times\left.\left(m_{\bar{K}^{* 0}}, M_{n}, m_{\bar{K}^{0}}\right) t_{\bar{K}^{0} n \rightarrow \pi^{0} \Sigma^{0}}\right|^{2} .
\end{aligned}
$$

\section{RESULTS}

Let us begin by showing in Fig. 5 the contribution of the triangle loop defined in Eq. (23). We plot the real and imaginary parts of $t_{T}\left(m_{K^{*-}}, M_{p}, m_{K^{-}}\right)$, as well as the 


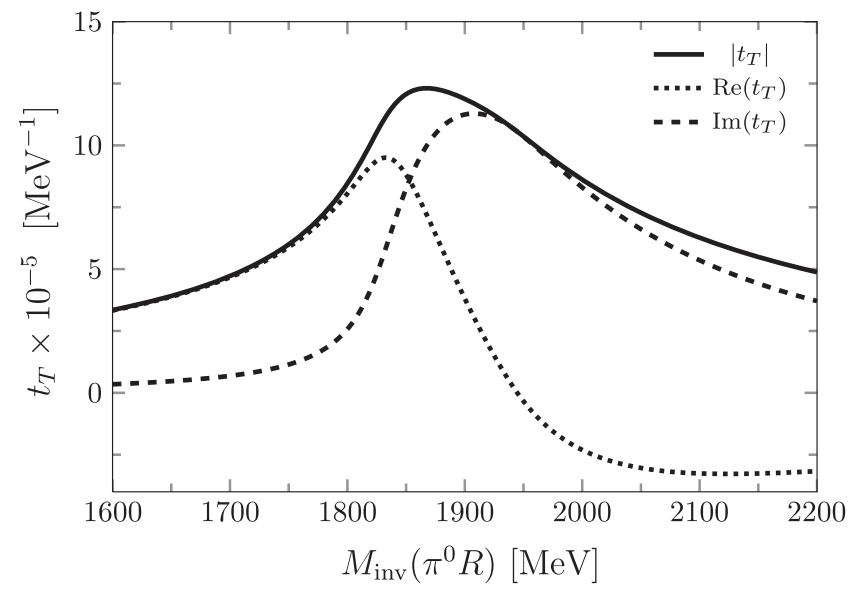

FIG. 5. Triangle amplitude $t_{T}$ for the decay in Fig. 1(a), here taking $M_{\text {inv }}(R)=1420 \mathrm{MeV}$.

absolute value with $M_{\text {inv }}(R) \equiv M_{\text {inv }}\left(\pi^{0} \Sigma^{0}\right)$ fixed at $1420 \mathrm{MeV}$. It can be observed that $\operatorname{Re}\left(t_{T}\right)$ has a peak around $1838 \mathrm{MeV}, \operatorname{Im}\left(t_{T}\right)$ has a peak around $1908 \mathrm{MeV}$, and there is a peak for $\left|t_{T}\right|$ around $1868 \mathrm{MeV}$. As discussed in Ref. [27], the peak of the real part is related to the $K^{*-} p$ threshold while the peak of the imaginary part, dominating for the larger invariant masses for $\pi^{0} R$, is due to the triangle singularity.

In Fig. 6 we plot the normalized double differential rate given by Eq. (28) for $\Lambda_{c}^{+} \rightarrow \pi^{+} \pi^{0} \pi^{0} \Sigma^{0}$ by fixing $M_{\text {inv }}\left(\pi^{0} R\right)=1850 \mathrm{MeV}, 1890 \mathrm{MeV}$, and $1930 \mathrm{MeV}$ and varying $M_{\text {inv }}(R)$. We can see that the distribution is highest near $M_{\text {inv }}\left(\pi^{0} R\right)=1890 \mathrm{MeV}$. For the three different masses of $M_{\text {inv }}\left(\pi^{0} R\right)$, we can also see a strong peak around $1432 \mathrm{MeV}$. Consequently, we see that most of the contribution to our width $\Gamma$ will come from $M_{\text {inv }}(R) \simeq M_{R}$, concretely in the range $M_{\text {inv }}\left(\pi^{0} \Sigma^{0}\right) \in[1390 \mathrm{MeV}, 1450 \mathrm{MeV}]$. The conclusion is that when we calculate the mass distribution $\frac{d \Gamma}{d M_{\text {inv }}\left(\pi^{0} \Lambda(1405)\right)}$, we can restrict the integral in $M_{\text {inv }}(R)$ $\left[M_{\text {inv }}\left(\pi^{0} \Sigma^{0}\right)\right.$ in Eq. (28)] to the limits mentioned above.

By integrating over $M_{\text {inv }}(R)$, we obtain $\frac{1}{\Gamma_{\Lambda_{c}^{+}}} \frac{d \Gamma}{d M_{\text {inv }}\left(\pi^{0} R\right)}$ which is shown in Fig. 7. We see a clear peak of the distribution around $1880 \mathrm{MeV}$ for $\Lambda(1405)$ production.

Integrating now $\frac{1}{\Gamma_{\Lambda^{+}}} \frac{d \Gamma}{d M_{\text {inv }}\left(\pi^{0} \Lambda(1405)\right)}$ over $M_{\text {inv }}\left(\pi^{0} \Lambda\right.$ $(1405)) \in[1800 \mathrm{MeV}, 2050 \mathrm{MeV}]$ in Fig. 7, we obtain the branching fraction

$$
\begin{aligned}
& \operatorname{Br}\left(\Lambda_{c}^{+} \rightarrow \pi^{+} \pi^{0} \Lambda(1405) ; \Lambda(1405) \rightarrow \pi^{0} \Sigma^{0}\right) \\
& \quad=(4.17 \pm 1.39) \times 10^{-6} .
\end{aligned}
$$

This number is within a measurable range. The errors come from the experimental errors in the branching ratio of $\operatorname{Br}\left(\Lambda_{c}^{+} \rightarrow \pi^{+} K^{*-} p\right)$.

One should stress the most remarkable feature in the distributions shown in Fig. 6: the width of the $\Lambda(1405)$
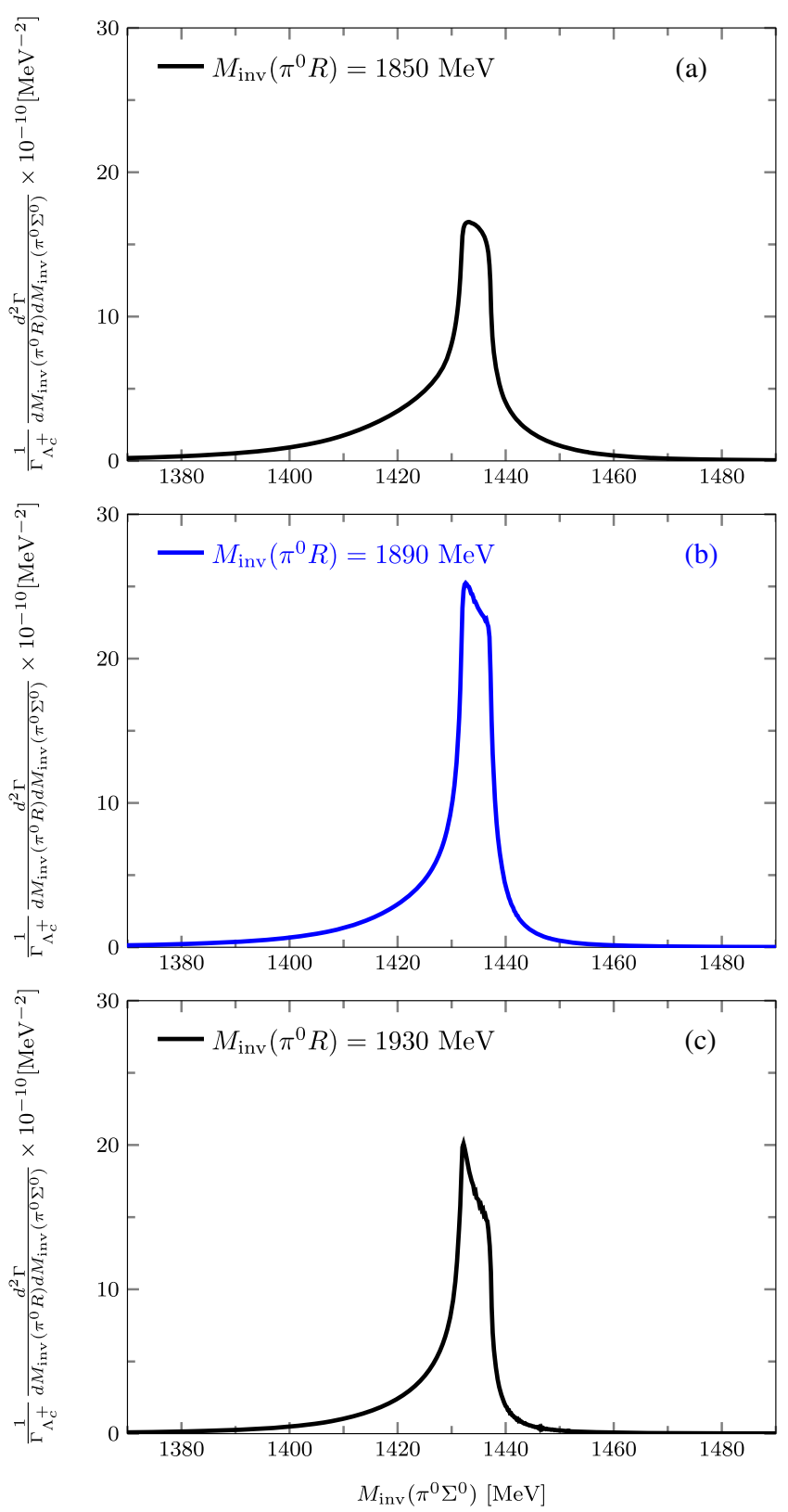

FIG. 6. Double differential width of $\Lambda_{c}^{+} \rightarrow \pi^{+} \pi^{0} \pi^{0} \Sigma^{0}$, keeping $M_{\text {inv }}\left(\pi^{0} R\right) \quad\left(R \equiv \pi^{0} \Sigma^{0}\right)$ fixed to three values, plotted versus $M_{\text {inv }}\left(\pi^{0} \Sigma^{0}\right)$. (a), (b), and (c) stand for $1850 \mathrm{MeV}, 1890 \mathrm{MeV}$ and $1930 \mathrm{MeV}$, respectively.

produced is a mere $6.5 \mathrm{MeV}$, remarkably smaller than the nominal widths for the $\Lambda(1405)$ at $1420 \mathrm{MeV}$ of the order of $30 \mathrm{MeV}$. As mentioned before, this narrow width is tied basically to the different masses of the $K^{-}, \bar{K}^{0}$ or $p, n$. This exceptionally narrow shape has been observed in all the isospin forbidden $f_{0}(980)$ production mode. The present reaction would be the first one where the narrow $\Lambda(1405)$ is seen in an isospin forbidden mode.

In order to see which are the most important ingredients in the isospin violation, in Fig. 8 we separate the effects. First we show the result for Fig. 6(b) where we introduce 


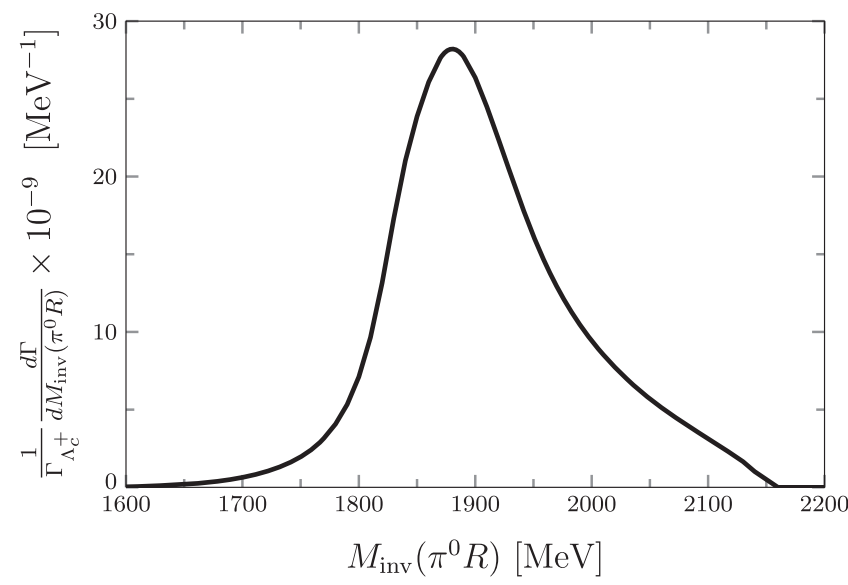

FIG. 7. The mass distribution of $\Lambda_{c}^{+} \rightarrow \pi^{+} \pi^{0} \pi^{0} \Sigma^{0}$ as a function of $M_{\text {inv }}\left(\pi^{0} R\right)$ with $R \equiv \pi^{0} \Sigma^{0}$. isospin violation in the scattering matrix elements $t_{K^{-}} p \rightarrow \pi^{0} \Sigma^{0}$, $t_{\bar{K}^{0} n \rightarrow \pi^{0} \Sigma^{0}}$, by doing the calculation of Ref. [38] with the actual masses of all the particles, but keeping $m_{\bar{K}^{0}}=m_{K^{-}}$ and $M_{p}=M_{n}$ in the triangle loop. Next we assume isospin symmetry in these amplitudes by performing the calculation with average masses of particles within the same multiplet and take the physical masses for $p, n, K^{-}, \bar{K}^{0}$ in the triangle loop. Third, we take the scattering amplitudes isospin symmetric and keep $m_{\bar{K}^{0}}=m_{K^{-}}$while considering actual masses for $p$ and $n$ in the triangle loop. Finally, we take the $t_{K^{-} p \rightarrow \pi^{0} \Sigma^{0}}, t_{\bar{K}^{0} n \rightarrow \pi^{0} \Sigma^{0}}$ isospin symmetric, and take $M_{p}=M_{n}$ but use the physical masses of $m_{K^{-}}$and $m_{\bar{K}^{0}}$ in the triangle loop. The masses of the $K^{*-}$ and $\bar{K}^{* 0}$ are reasonably similar and we take the same for the two. By comparing with Fig. 6(b) we see that the most important ingredient in the isospin violation comes from the $\bar{K}^{0}$ and $K^{-}$mass difference. In Fig. 8, the solid line refers to Fig. 6(b).
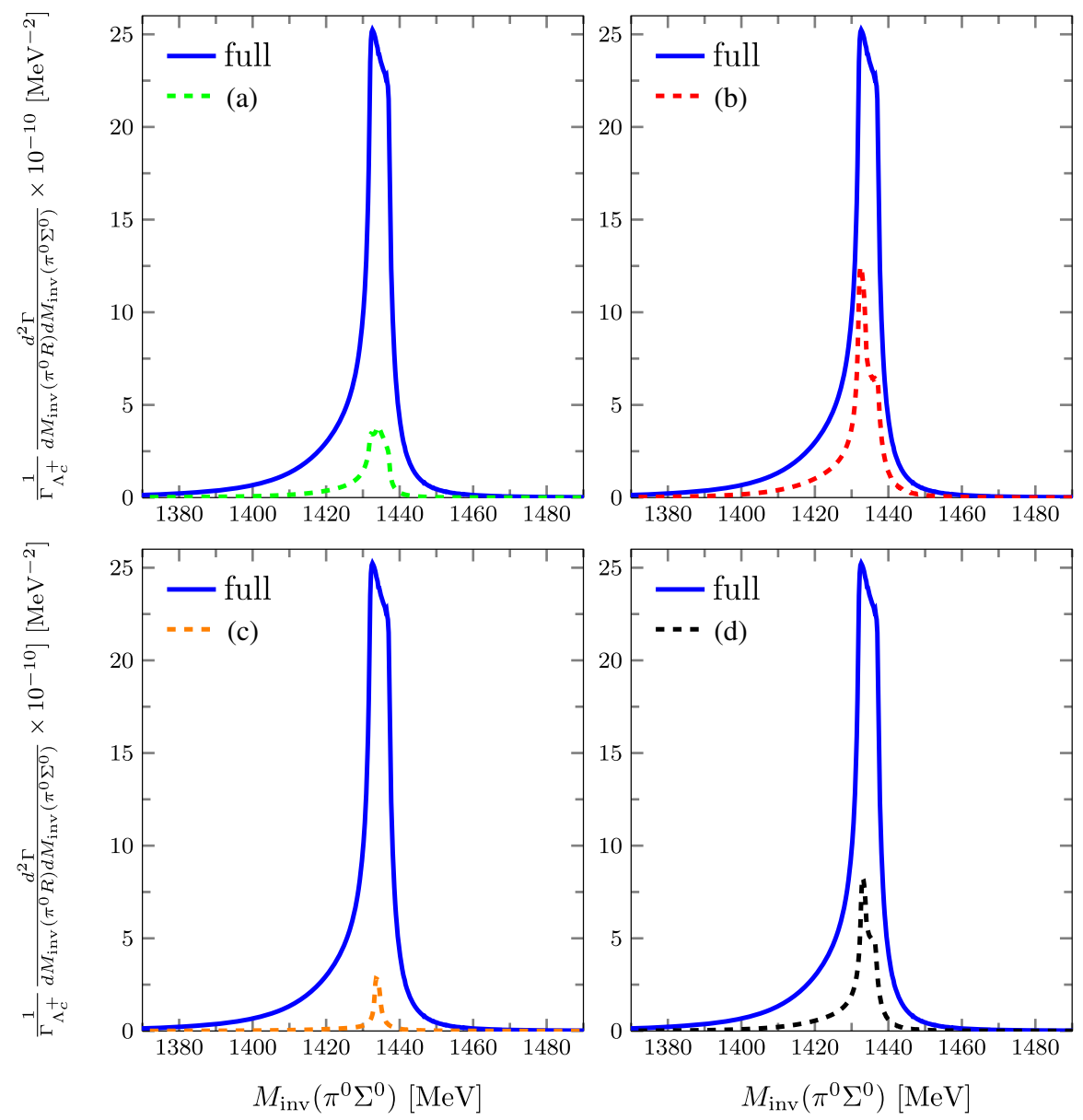

FIG. 8. Double differential width of $\Lambda_{c}^{+} \rightarrow \pi^{+} \pi^{0} \pi^{0} \Sigma^{0}$, keeping $M_{\text {inv }}\left(\pi^{0} R\right)\left(R \equiv \pi^{0} \Sigma^{0}\right)$ fixed at $1890 \mathrm{MeV}$ as a function of $M_{\text {inv }}\left(\pi^{0} \Sigma^{0}\right)$, where full shows the result for Fig. 6(b); (a) corresponds to taking $t_{K^{-}} p \rightarrow \pi^{0} \Sigma^{0}, t_{\bar{K}^{0} n \rightarrow \pi^{0} \Sigma^{0}}$ with the physical masses of the particles but $M_{p}=M_{n}, m_{\bar{K}^{0}}=m_{K^{-}}$; (b) corresponds to taking $t_{K^{-} p \rightarrow \pi^{0} \Sigma^{0}}, t_{\bar{K}^{0} n \rightarrow \pi^{0} \Sigma^{0}}$ isospin symmetric (equal) and physical masses for $p, n, K^{-}, \bar{K}^{0}$; (c) stands for the scattering amplitude isospin symmetric, $m_{\bar{K}^{0}}=m_{K^{-}}$, but $M_{p}$ and $M_{n}$ with their physical values. (d) stands for the scattering amplitude isospin symmetric, $M_{p}=M_{n}$, but $m_{\bar{K}^{0}}$ and $m_{K^{-}}$with their physical values. 


\section{CONCLUSIONS}

The triangle singularities have shown to be very effective, enhancing the production of $f_{0}(980)$ or $a_{0}(980)$ in isospin suppressed modes. These reactions have played a double role. On the first hand, they have provided clear examples of triangle singularities, and on the second hand, the rates and shapes obtained for the isospin suppressed modes are closely tied to the nature of these resonances and offer extra support to their dynamical origin from the interaction of mesons in coupled channels.

The two states of the $\Lambda(1405)$, now already official in the PDG, are another example of dynamical generation from the interaction of meson baryon in this case. Yet, the resonance has not been observed in an isospin-violating reaction so far. The present work provides the first evaluation of the $\Lambda(1405)$ production in an isospin forbidden reaction. We devised one such reaction, which, as in the case of the $f_{0}(980)$ or $a_{0}(980)$, can be enhanced by a triangle singularity. We found such an example in the decay of $\Lambda_{c}^{+}$into $\pi^{+} \pi^{0} \Lambda(1405)$. The mechanism for the production is given by a first decay of the $\Lambda_{c}^{+}$into $\pi^{+} \bar{K}^{*} N$, and then the $\bar{K}^{*}$ decays into $\bar{K} \pi$ and the $\bar{K} N$ merge to produce the $\Lambda(1405)$ through a triangle loop containing $\bar{K}^{*} N \bar{K}$, which develops a singularity around $1890 \mathrm{MeV}$.

The remarkable observation is that a peak tied to the $\Lambda(1405)$ state of higher energy (around $1420 \mathrm{MeV}$ ) appears in the final $\pi^{0} \Sigma^{0}$ mass spectrum, but peaking even at higher energy, close to the $\bar{K} N$ threshold of $1432 \mathrm{MeV}$. It is also remarkably narrow, of the order of $6-7 \mathrm{MeV}$, and is tied to the difference of masses between the $K^{-}$and $\bar{K}^{0}$ and $p, n$, but mostly to the $K^{-}$and $\bar{K}^{0}$ mass difference, although there is also a small effect from isospin violation in the $t_{K^{-} p \rightarrow \pi^{0} \Sigma^{0}}$ and $t_{\bar{K}^{0} n \rightarrow \pi^{0} \Sigma^{0}}$ amplitudes.

We have shown that the amount of $\Lambda(1405)$ production has its largest strength at a $\pi^{0} \Lambda(1405)$ invariant mass of around $1890 \mathrm{MeV}$, where the mechanism suggested develops a triangle singularity. The shape and strength obtained are intimately tied to the nature of the $\Lambda(1405)$ as a dynamically generated resonance from the meson baryon interaction, and in the present case, to its large coupling to the $\bar{K} N$ component. We found that the strength of the calculated decay width falls within a measurable range. The implementation of the reaction would thus bring valuable information on the nature of this resonance, the mechanisms of triangle singularities, plus extra information on the continuously searched for $\bar{K} N$ interaction.

\section{ACKNOWLEDGMENTS}

We are very thankful to the referee for many suggestions. L. R. D. acknowledges the support from the National Natural Science Foundation of China (No. 11575076) and the State Scholarship Fund of China (No. 201708210057). R. P. P. thanks the Generalitat Valenciana in the program Santiago Grisolia. This work is partly supported by the Spanish Ministerio de Economia y Competitividad and European FEDER funds under Contracts No. FIS2011-28853-C02-01, No. FIS2011-28853-C02-02, No. FIS2014-57026-REDT, No. FIS2014-51948-C2-1-P, and No. FIS2014-51948-C22-P, and the Generalitat Valenciana in the program Prometeo II-2014/068 (EO).
[1] L. D. Landau, Nucl. Phys. 13, 181 (1959).

[2] S. Coleman and R. E. Norton, Nuovo Cimento 38, 438 (1965).

[3] M. Bayar, F. Aceti, F. K. Guo, and E. Oset, Phys. Rev. D 94, 074039 (2016).

[4] M. Mikhasenko, B. Ketzer, and A. Sarantsev, Phys. Rev. D 91, 094015 (2015).

[5] X. H. Liu, M. Oka, and Q. Zhao, Phys. Lett. B 753, 297 (2016).

[6] F. Aceti, L. R. Dai, and E. Oset, Phys. Rev. D 94, 096015 (2016).

[7] C. Adolph et al. (COMPASS Collaboration), Phys. Rev. Lett. 115, 082001 (2015).

[8] L. Roca, E. Oset, and J. Singh, Phys. Rev. D 72, 014002 (2005).

[9] M. F. M. Lutz and E. E. Kolomeitsev, Nucl. Phys. A730, 392 (2004).

[10] Y. Zhou, X. L. Ren, H. X. Chen, and L. S. Geng, Phys. Rev. D 90, 014020 (2014).
[11] J. A. Oller and E. Oset, Nucl. Phys. A620, 438 (1997); A652, 407(E) (1999).

[12] M. P. Locher, V. E. Markushin, and H. Q. Zheng, Eur. Phys. J. C 4, 317 (1998).

[13] N. Kaiser, Eur. Phys. J. A 3, 307 (1998).

[14] J. Nieves and E. Ruiz Arriola, Nucl. Phys. A679, 57 (2000).

[15] D. Gamermann, E. Oset, D. Strottman, and M. J. V. Vacas, Phys. Rev. D 76, 074016 (2007).

[16] W. H. Liang and E. Oset, Phys. Lett. B 737, 70 (2014).

[17] J. J. Xie, L. R. Dai, and E. Oset, Phys. Lett. B 742, 363 (2015).

[18] F. K. Guo, C. Hanhart, U. G. Meißner, Q. Wang, Q. Zhao, and B. S. Zou, Rev. Mod. Phys. 90, 015004 (2018).

[19] C. Patrignani et al. (Particle Data Group), Chin. Phys. C 40, 100001 (2016).

[20] V. R. Debastiani, F. Aceti, W. H. Liang, and E. Oset, Phys. Rev. D 95, 034015 (2017).

[21] J. J. Xie, L. S. Geng, and E. Oset, Phys. Rev. D 95, 034004 (2017). 
[22] A. P. Szczepaniak, Phys. Lett. B 747, 410 (2015).

[23] A. E. Bondar and M. B. Voloshin, Phys. Rev. D 93, 094008 (2016).

[24] A. Pilloni, C. Fernández-Ramírez, A. Jackura, V. Mathieu, M. Mikhasenko, J. Nys, and A.P. Szczepaniak (JPAC Collaboration), Phys. Lett. B 772, 200 (2017).

[25] R. Pavao, S. Sakai, and E. Oset, Eur. Phys. J. C 77, 599 (2017).

[26] X. H. Liu and U. G. Meißner, Eur. Phys. J. C 77, 816 (2017).

[27] S. Sakai, E. Oset, and A. Ramos, Eur. Phys. J. A 54, 10 (2018).

[28] J. J. Wu, X. H. Liu, Q. Zhao, and B. S. Zou, Phys. Rev. Lett. 108, 081803 (2012).

[29] F. Aceti, W. H. Liang, E. Oset, J. J. Wu, and B. S. Zou, Phys. Rev. D 86, 114007 (2012).

[30] X. G. Wu, J. J. Wu, Q. Zhao, and B. S. Zou, Phys. Rev. D 87, 014023 (2013).

[31] M. Ablikim et al. (BESIII Collaboration), Phys. Rev. Lett. 108, 182001 (2012).

[32] S. Sakai, E. Oset, and W. H. Liang, Phys. Rev. D 96, 074025 (2017).

[33] W. H. Liang, S. Sakai, J. J. Xie, and E. Oset, Chin. Phys. C 42, 044101 (2018).

[34] R. H. Dalitz and S. F. Tuan, Phys. Rev. Lett. 2, 425 (1959).

[35] R. H. Dalitz and S. F. Tuan, Ann. Phys. (N.Y.) 10, 307 (1960).

[36] R. H. Dalitz, T. C. Wong, and G. Rajasekaran, Phys. Rev. 153, 1617 (1967).

[37] N. Kaiser, P. B. Siegel, and W. Weise, Nucl. Phys. A594, 325 (1995).

[38] E. Oset and A. Ramos, Nucl. Phys. A635, 99 (1998).

[39] J. A. Oller and U. G. Meißner, Phys. Lett. B 500, 263 (2001).

[40] M. F. M. Lutz and E. E. Kolomeitsev, Nucl. Phys. A700, 193 (2002).

[41] C. Garcia-Recio, J. Nieves, E. R. Arriola, and M. J. V. Vacas, Phys. Rev. D 67, 076009 (2003).

[42] D. Jido, J. A. Oller, E. Oset, A. Ramos, and U. G. Meißner, Nucl. Phys. A725, 181 (2003).
[43] T. Hyodo and D. Jido, Prog. Part. Nucl. Phys. 67, 55 (2012).

[44] Y. Kamiya, K. Miyahara, S. Ohnishi, Y. Ikeda, T. Hyodo, E. Oset, and W. Weise, Nucl. Phys. A954, 41 (2016).

[45] E. Oset et al., Int. J. Mod. Phys. E 25, 1630001 (2016).

[46] T. Hyodo and M. Oka, Phys. Rev. C 84, 035201 (2011).

[47] K. Miyahara, T. Hyodo, and E. Oset, Phys. Rev. C 92, 055204 (2015).

[48] L. J. Liu, E. Wang, J. J. Xie, K. L. Song, and J. Y. Zhu, arXiv:1712.07469.

[49] K. Miyahara and T. Hyodo, arXiv:1803.05572.

[50] E. Wang, J. J. Xie, W. H. Liang, F. K. Guo, and E. Oset, Phys. Rev. C 95, 015205 (2017).

[51] J. J. Xie, W. H. Liang, and E. Oset, Phys. Lett. B 777, 447 (2018).

[52] M. Bayar, R. Pavao, S. Sakai, and E. Oset, Phys. Rev. C 97 , 035203 (2018).

[53] F. Mandl and G. Shaw, Quantum Field Theory (John Wiley \& Sons, New York, 1984)

[54] L. L. Chau, Phys. Rep. 95, 1 (1983).

[55] K. Miyahara, T. Hyodo, M. Oka, J. Nieves, and E. Oset, Phys. Rev. C 95, 035212 (2017).

[56] M. Bando, T. Kugo, and K. Yamawaki, Phys. Rep. 164, 217 (1988).

[57] U. G. Meißner, Phys. Rep. 161, 213 (1988).

[58] H. Nagahiro, L. Roca, A. Hosaka, and E. Oset, Phys. Rev. D 79, 014015 (2009).

[59] M. Bando, T. Kugo, S. Uehara, K. Yamawaki, and T. Yanagida, Phys. Rev. Lett. 54, 1215 (1985).

[60] A. Bramon, A. Grau, and G. Pancheri, Phys. Lett. B 283, 416 (1992).

[61] F. Aceti, J. M. Dias, and E. Oset, Eur. Phys. J. A 51, 48 (2015).

[62] N. N. Achasov, S. A. Devyanin, and G. N. Shestakov, Phys. Lett. 88B, 367 (1979).

[63] C. Hanhart, Phys. Rep. 397, 155 (2004).

[64] C. Hanhart, B. Kubis, and J. R. Pelaez, Phys. Rev. D 76, 074028 (2007).

[65] L. Roca, Phys. Rev. D 88, 014045 (2013). 